\title{
Reynolds number effects on flow/acoustic mechanisms in spherical windscreens ${ }^{\text {a) }}$
}

\author{
Z. Charlie Zheng ${ }^{\text {b) }}$ and Bee K. Tan \\ Department of Mechanical and Nuclear Engineering, Kansas State University, Manhattan, Kansas 66506
}

(Received 5 April 2002; revised 12 September 2002; accepted 24 September 2002)

\begin{abstract}
There is a practical need to fully understand the mechanisms involved in the flow/pressure fluctuations around a screened microphone. A stream of uniform flow with low-frequency turbulence encountering a rigid, impermeable spherical windscreen is considered in this study. Pressure distributions on the surface of the sphere are determined by the flow structure. Pressure fluctuations at the center of the sphere are then calculated based on the integration of surface pressure distributions. Because of the low-frequency assumption, results from steady-state laminar flows can be used to investigate the Reynolds number effects on wind noise reduction. Three types of flow have been studied in this paper: an inviscid case, a low-Reynolds-number Stokes flow, and intermediate- and high-Reynolds-number flows. A Reynolds-number/wind-noise-reduction correlation shows that the wind noise reduction increases with decreasing Reynolds number. (C) 2003 Acoustical Society of America. [DOI: 10.1121/1.1527927]
\end{abstract}

PACS numbers: 43.28.Gq, 43.28.Ra, 43.50.Gf, 43.28.Hr [MSH]

\section{INTRODUCTION}

Windscreens are widely used in outdoor microphone measurement; therefore, there is a practical need to fully understand the mechanisms involved in the flow/pressure fluctuations around a screened microphone. The understanding may lead to optimized design of the windscreen for measurement microphones. However, the wind noise sensed by a microphone inside a windscreen is a complicated aerodynamic noise problem. In low-turbulence flows, the dominant noise is produced by the interaction of the flow with the microphone as a result of pressure gradients across the microphone face due to wake shedding from the microphone. Strasberg (1988) performed dimensional analysis of windscreen noise for measurements made in low-turbulence conditions and showed that the one-third-octave sound pressure obeyed scaling laws involving the average speed of the flow, the air density, and the diameter of the screen. This suggests that windscreen-generated velocity fluctuations account for the wind noise in low-turbulence environments. Morgan and Raspet (1992) argued that under high-turbulence conditions, the dominant source of pressure fluctuations at the microphone outdoors was the intrinsic turbulence in the incoming flow. For flow through a porous windscreen or over a rough foam surface, the amplitude of the pressure fluctuation can possibly be reduced due to reduced wake fluctuations, as shown by Hosier and Donavan (1979) (although under the low-turbulence conditions in their study).

In this study, analytical methods have been used to investigate the flow/acoustic mechanisms assuming a highturbulence scenario. Therefore, the vortex shedding effects

\footnotetext{
${ }^{\text {a)} P o r t i o n s ~ o f ~ t h i s ~ w o r k ~ w e r e ~ p r e s e n t e d ~ i n ~ Z . ~ C . ~ Z h e n g, ~ " A ~ c o m p u t a t i o n a l ~}$ study of the flow/acoustic mechanisms in screened microphones," Proceedings of ASME FEDSM'01, FEDSM2001-18204, 2001 ASME Fluids Engineering Division Summer Meeting, 29 May-1 June 2001, New Orleans, LA.

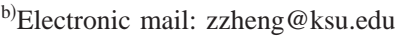

are not considered. A rigid surface, impermeable spherical windscreen model is used to study wind noise reductions at low frequencies. In this model, the impermeable surface of the windscreen transmits the pressure fluctuations to quiescent air media inside. Since the turbulence outdoors is highly weighted to low frequencies, the largest contributions to the overall wind noise level are at low frequencies. Therefore, the study is restricted to wind noise generation due to turbulence whose spatial scales are much larger than the windscreen diameter. The mean flow across the screen can be considered as a steady flow. The results of this model have given good agreement in comparison with experimental data in literature. The model could thus be adopted for investigating the theoretical calculation of wind noise reduction (WNR) in more complicated windscreens such as porous medium windscreens.

To justify the use of a steady-state flow model, two sets of measurement data by Morgan (1993) for WNR versus screen numbers are plotted in Fig. 1. The screen number $(D / \lambda)$ is defined as the ratio between the windscreen diameter, $D$, and the wavelength, $\lambda$. The first set of data is for a windscreen of $90-\mathrm{mm}$ diameter in a flow of $4.85 \mathrm{~m} / \mathrm{s}$, and the second set is for a windscreen of $180-\mathrm{mm}$ diameter in a flow of $4.74 \mathrm{~m} / \mathrm{s}$. It can be seen that when the screen number is below 0.3 , the WNR is almost constant. After that, the WNR increases rapidly with an increase in the screen number. This is because the scale of the turbulence becomes smaller and the steady-state assumption is no longer valid. Figure 1 also shows that the analysis presented in this paper only applies to the cases where the length scale of the turbulence is larger than the size of the windscreen.

The choice of a simple, steady-state system makes it possible to compare the calculated results to measurements of pressure coefficients on smooth rigid spheres and to other analytical models. The pressure fluctuations induced on the spherical windscreen surface can then be used to obtain the 


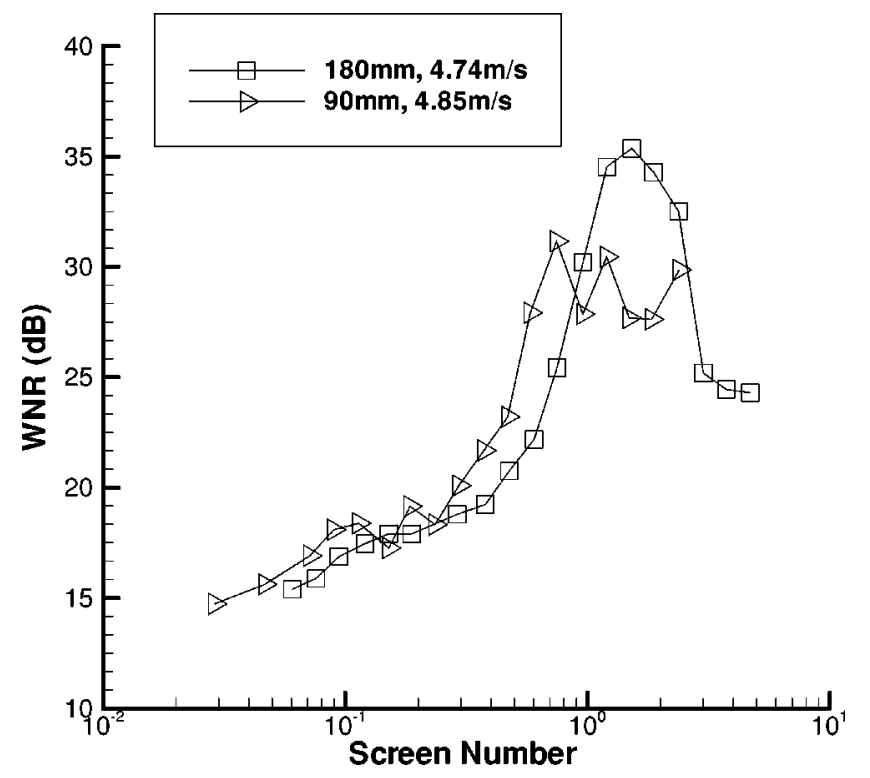

FIG. 1. Measured wind noise reduction by Morgan (1993), as a function of screen number $(D / \lambda)$, where $D$ is the diameter of the windscreen and $\lambda$ is the wavelength. The data are extracted from Fig. 14 in Morgan (1993). The triangle-symboled line is for a windscreen with diameter of $90 \mathrm{~mm}$ and flow speed of $4.84 \mathrm{~m} / \mathrm{s}$. The square-symboled line is for a windscreen with diameter of $180 \mathrm{~mm}$ and flow speed of $4.85 \mathrm{~m} / \mathrm{s}$.

pressure levels which can be sensed by a microphone placed at the center of the windscreen.

In the following sections, the pressure fluctuation relationship inside and outside on the surfaces of the sphere is presented at first. The results are then used to study three types of flow: an inviscid case, a low-Reynolds-number Stokes flow, and intermediate- and high-Reynolds-number flows. The inviscid and Stokes flow cases are the two extreme cases in which analytical solutions can be pursued. The flow field of the third case can be calculated using computational fluid dynamics (CFD) techniques. A flow solver software, FLUENT, was used to provide flow field to determine the pressure distribution on the sphere surface. The wind noise reduction on the microphone can be determined from both the theoretical results and experimental data in literature, using the steady-state pressure distribution on the surface. Finally, a correlation between the WNR and Reynolds numbers is obtained.

\section{PRESSURE FLUCTUATION RELATIONSHIPS}

It is assumed that there is no flow inside the windscreen sphere. Therefore, the pressure fluctuations inside the sphere obey the Laplace equation under the incompressibility assumption. Theoretically, the pressure inside the sphere can thus be fully determined by the surface pressure. However, the validity and accuracy of using surface pressure integrations to estimate the center pressure for the windscreen noise reduction calculation, which is based on the solution of the Laplace equation, are unable to be fully addressed at this point. This requires that the results presented here be compared with those from the simulations with permeable boundaries on the sphere. The only indication of validity of the proposed idealized treatment is the results from Morgan's (1993) measurement, where the integration of measured sur- face pressure coefficient, $C_{p}$, gave values close to the measured wind noise reduction levels at the center of the screen.

In a spherical coordinate system, $(r, \theta, \phi)$, where $\phi$ is the azimuthal angle, the average value property of the Laplace equation yields the following solution for the pressure at the center of the sphere:

$$
p(0)=\frac{1}{4 \pi} \int_{0}^{2 \pi} d \phi \int_{0}^{\pi} p(a, \theta, \phi) \sin \theta d \theta .
$$

This means that the wind noise detected at the center of the screen is the average of the surface pressure fluctuations.

If we assume azimuthal symmetry in this spherical problem, i.e., $p(a, \theta, \phi)$ independent of $\phi$, Eq. (1) reduces to

$$
p(0)=\frac{1}{2} \int_{0}^{\pi} p(a, \theta) \sin \theta d \theta .
$$

Consequently, if we let

$$
x=\cos \theta,
$$

and

$$
p(a, \theta)=f(x),
$$

then we can have

$$
p(0)=\frac{1}{2} \int_{-1}^{1} f(x) d x .
$$

It is worth noting that general solutions for pressure fluctuations at locations other than the center under nonaxisymmetric outside flow field can be obtained by using spherical harmonics following a similar procedure to that in Jackson (1963).

\section{INVISCID CASE}

For unsteady, inviscid incompressible flow over a sphere, the flow is irrotational if the incoming flow is uniform. A three-dimensional velocity potential exists for flow around a sphere (e.g., Currie, 1993), as follows:

$$
\phi(r, \theta)=U\left(r+\frac{1}{2} \frac{a^{3}}{r^{2}}\right) \cos \theta,
$$

where $U$ is the incoming velocity. Using the unsteady Bernoulli equation, Phelps (1938) showed that on the surface of a sphere

$$
p(a, \theta)=\frac{1}{8} \rho U^{2}\left(9 \cos ^{2} \theta-5\right)+\frac{3}{2} \rho a\left(\frac{\partial U}{\partial t}\right) \cos \theta+P,
$$

where $P$ is the pressure of the incoming flow. The velocity, $U$, can be expressed as

$$
U=\bar{U}+u^{\prime} .
$$

The prime for the pressure fluctuations in this paper is omitted. If only the pressure fluctuations caused by flow are considered, and neglecting higher order terms, Eq. (7) yields

$$
\frac{p(a, \theta)}{\rho \bar{U} u^{\prime}}=\frac{1}{4}\left[9 \cos ^{2} \theta-5\right]+\frac{3}{2} \frac{a}{\bar{U} u^{\prime}} \frac{\partial u^{\prime}}{\partial t} \cos \theta .
$$


Once the surface pressure fluctuation is known, Eq. (5) can be used to obtain the pressure fluctuation at the sphere center. Since the integration of the odd function terms of $x$ in $f(x)$ must be zero, the effects of the second term in Eq. (9) vanish. Therefore

$$
\frac{p(0)}{\rho \bar{U} u^{\prime}}=\frac{1}{2} \int_{-1}^{+1} g(x) d x=-\frac{1}{2},
$$

where

$$
g(x)=\frac{1}{4}\left[9 x^{2}-5\right] .
$$

The integral function, $g(x)$, is the same as the pressure distribution coefficient, $C_{p}$, on a sphere in steady flow. It should be noted that this pressure distribution coefficient, which is symmetric with respect to $x=0$, results in zero drag (D'Alembert paradox). However, the arithmetic average of it, Eq. (10), results in a net value of $-1 / 2$. Since the unscreened pressure fluctuation is $\rho \bar{U} u^{\prime}$ in a uniform stream, the wind noise reduction of the spherical screen in decibels is

$$
\mathrm{WNR}=-10 \log _{10}\left(\frac{p(0)}{\rho \bar{U} u^{\prime}}\right)^{2}=6.02 \mathrm{~dB} .
$$

According to the above procedure, the WNR of a spherical screen is determined by the integration of the surface $C_{p}$ from a corresponding steady flow. This concept will be extended to finite Reynolds-number flows later in the paper, where experiment and CFD results from steady-state studies will be used to determine the wind noise reduction. Apparently, the wind noise reduction is independent of the velocity fluctuation frequency as well as the windscreen size. This is caused by two factors: the inviscid assumption and the microphone being at the center of the sphere. However, for low-frequency turbulence, the frequency of incoming velocity fluctuations is low (in comparison with $\bar{U} / a$ ); thus, the second term on the right-hand side of Eq. (9) can be neglected. The surface pressure fluctuation is then independent of the size of the screen. Therefore, under conditions of lowfrequency turbulence, the wind noise reduction does not change with the size of the screen. Furthermore, this is true even when the microphone is not located at the center of the sphere, because the pressure inside the sphere is determined by the surface pressure only.

Thus far, the analysis presented in this section has been for an unsteady flow in which the fluctuation flow $\left(u^{\prime}\right)$ was parallel to the mean flow $(\bar{U})$. During the review process associated with this article, it was brought to the attention of the authors that forcing the velocity fluctuation to be in parallel with the mean velocity was in fact nonphysical. Alternatively, it can be shown that, since the pressure fluctuation is related to the linear term in the square of velocity magnitude, the contributions from velocity fluctuations perpendicular to the mean flow are of higher order, according to the following expression:

$$
\begin{aligned}
|\mathbf{U}|^{2} & =\left|\bar{U} \mathbf{i}+u^{\prime} \mathbf{i}+v^{\prime} \mathbf{j}+w^{\prime} \mathbf{k}\right|^{2} \\
& =\bar{U}^{2}+2 \bar{U} u^{\prime}+u^{\prime 2}+v^{\prime 2}+w^{\prime 2}
\end{aligned}
$$

Therefore, the results are still correct even with an arbitrary velocity fluctuation.

\section{LOW-REYNOLDS-NUMBER FLOW}

The Stokes creeping-flow approximation is used in this case. Although this flow may not actually occur in practice for the problem discussed here, it is analyzed for the purpose of comparison. The pressure fluctuation on the surface is expressed as (e.g., Currie, 1993)

$$
\frac{p(a, \theta)}{\rho \bar{U} u^{\prime}}=-\frac{3}{2} \frac{\cos \theta}{\operatorname{Re}},
$$

where

$$
\operatorname{Re}=\frac{\rho \bar{U} a}{\mu} .
$$

Because of the antisymmetric pressure distribution with respect to $x=0$ in Eq. (13), the pressure fluctuation at the center, which is the arithmetic average of the distribution, is thus equal to zero. This means for a low-Reynolds-number flow, the wind noise due to flow fluctuation can be totally screened out if the microphone is placed at the center.

By comparing the cases in Secs. III and IV, it can be seen that the wind noise reduction is reduced from infinite for very low Reynolds-number flow to $6.02 \mathrm{~dB}$ for inviscid flow (with infinite Reynolds numbers). The surface pressure distribution changes from antisymmetric to symmetric with respect to $x=0 \quad(\theta=\pi / 2)$ when the Reynolds number increases, with the lower bound of the wind noise reduction occurring in the symmetric pressure distribution case. In the next section, high but finite Reynolds number cases are considered which are more applicable in realistic situations. The wind noise reduction is expected to fall between infinity and $6.02 \mathrm{~dB}$, and the pressure distributions on the surface of the sphere should be neither symmetric nor antisymmetric with respect to the $x$ direction.

\section{INTERMEDIATE- AND HIGH-REYNOLDS-NUMBER FLOW}

For intermediate or high Reynolds numbers, analytical solutions do not exist and the analysis has to be based on experimental data or numerical solutions. In this study, computational fluid dynamics is used to compute the flow field, and comparisons are made with well-known experimental data. Because the typical turbulence eddy size is much larger than the screen radius, the computations are mostly based on a laminar flow model. Turbulent simulations are also performed for comparison purposes. A flow solver software, FLUENT, is used to solve the three-dimensional NavierStokes equations. The pressure distributions on the spherical surface can be obtained from the CFD solution. It has been discussed earlier that for low-frequency turbulence, the distribution function of the surface pressure fluctuations is the same as that of the steady pressure distribution. Therefore, a steady-state computation is used to determine the distribution 

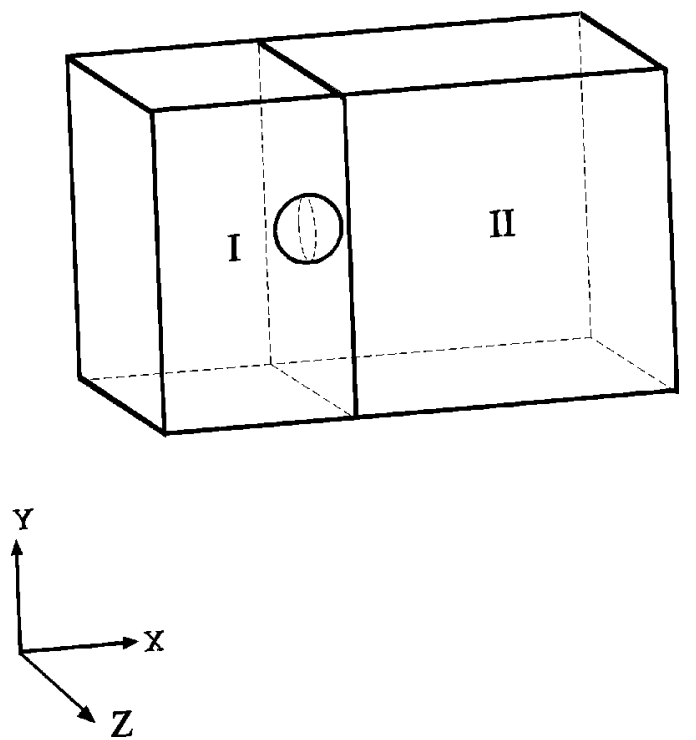

FIG. 2. The computational fluid dynamics model, where the incoming flow is in the positive $x$ direction. Volumes I and II are the two volumes used to map the grid mesh.

function. The integration of the distribution function, as shown in Eq. (5), yields the pressure fluctuation at the sphere center.

The computational model is shown in Fig. 2. The computational domain is a $16 a$ (in the $x$ direction) $\times 10 a \times 10 a$ box, with a sphere of radius $a$ inside. The center of the sphere is located a distance of $6 a$ downstream from the inflow plane in the flow direction, which is also the $x$ direction. The sphere is also centered in the computational domain with respect to the $y$ - and $z$ coordinates. A uniform velocity in the $x$ direction is specified at the inflow plane. The error introduced by this can be estimated using Eq. (6), which shows that the influence of the sphere on the inflow velocity decays at $O\left(a^{3} / r^{3}\right)$. Hence, less than $0.5 \%\left[O\left(1 / 6^{3}\right)\right]$ velocity straining at the in-flow plane is introduced by placing a sphere centered a distance of $6 a$ downstream from the inflow plane. Lee (2000) chose an upstream in-flow plane at $6 a$ for lower Reynolds number cases, with $\mathrm{Re}$ in the range of $O\left(10^{2}\right)$. It was noticed that the influence of the sphere on the inflow was more significant at lower Reynolds numbers. From the velocity distribution in Stokes flow past a sphere, the influence of the sphere decays with distance according to $O(a / r)$. Therefore, the selection of the uniform inflow plane position for the given Reynolds number range is sufficiently upstream from the sphere. At the outflow plane, an outflow extrapolation boundary condition is specified. Free-stream boundary conditions are specified on the side boundaries of the computational box. Since the side walls are $5 a$ away from the sphere center, the side-wall boundary effects on the flow field near the sphere surface can be neglected.

A grid generation package, GAMBIT, was used to generate the numerical grid. The domain was split into two subdomains, volume I and volume II, with a $y-z$ junction plane at the center of the sphere (at $x=0$ ), as shown in Fig. 2, in order to create a source face in each subdomain for permissible Cooper meshing. A grid number of 50 was used on all the edges in each volume. A grid interval ratio of 0.3 was used towards the junction plane of the two volumes in the $x$ direction. In the $y$ - and $z$ directions, a grid interval ratio of 0.8 was used towards the centers of the edges (double ratio). The purpose of these interval ratios was to generate a fine grid mesh near the sphere surface. The Cooper algorithm was then used to generate a hex/wedge grid mesh. It projected or extruded the boundary face mesh from one end of a volume to the other and then divided up the extruded mesh to form the volume mesh. After the projection, the number of grids on the surface of the sphere was 376 in the flow direction (the $x$ direction) and 32 in the azimuthal direction. The grid number was doubled to test the resolution requirement, and only minor differences in the results were found.

The grid mesh was then imported to FLUENT. Since only large-scale, low-frequency turbulence effects were considered, steady-state computations were performed, as discussed previously. A segregated steady-state 3D laminar solver was used with an implicitly iterative, second-order upwind scheme for momentum equations and the SIMPLE algorithm (Patankar, 1980) for pressure-velocity coupling. The convergence criteria were set at $10^{-4}$ for velocity residuals and $10^{-3}$ for continuity residual. Air was used as the simulation fluid, with $\rho=1.225 \mathrm{~kg} / \mathrm{m}^{3}$ and $\mu=1.789 \times 10^{-5} \mathrm{~kg} / \mathrm{m}-\mathrm{s}$. Values of inlet velocity were specified based on the Reynolds number range.

For a 45-mm-radius screen with incoming flow at 9.5 $\mathrm{m} / \mathrm{s}$, the Reynolds number is approximately $3 \times 10^{4}$. According to Achenbach's experiment (1972), the critical Reynolds number at which the drag coefficient reaches the minimum is $1.75 \times 10^{5}$. In addition, a transition in the free shear layer from a laminar to a turbulent flow occurs near the critical Reynolds number. Therefore, most of the windscreens operate within the subcritical Reynolds number range where the flow remains laminar after the separation, again justifying the usage of laminar simulations.

Turbulent cases were also added in the simulations for comparison purposes, and to provide additional data points to be used for subsequent curve fitting, although the very high Reynolds number cases are rare in windscreen problems. We chose the Spalart-Allmaras model (1992) for the turbulence, which is a relatively simple one-equation model that solves a modeled transport equation for the kinematic eddy (turbulent) viscosity. The Spalart-Allmaras model, designed especially for applications involving wall-bounded flows, has been shown to give good results for boundary layers subjected to adverse pressure gradients. An additional advantage is that in FLUENT, the Spalart-Allmaras model has been implemented to use wall functions when the mesh resolution does not need to be very fine. This might make it the best choice for relatively coarse meshes. Furthermore, the near-wall gradients of the transport variables in the model are much smaller than the gradients of the transport variables in $k-\epsilon$ type models. This also might make the model less sensitive to numerical errors when nonlayered meshes are used near walls, as in current simulations. However, higher resolutions are still required for these cases than for the laminar cases, especially near the surface of the sphere. The number of points on each edge was doubled to 100 , with grid interval ratios towards the junction plane of 0.25 in volume I 

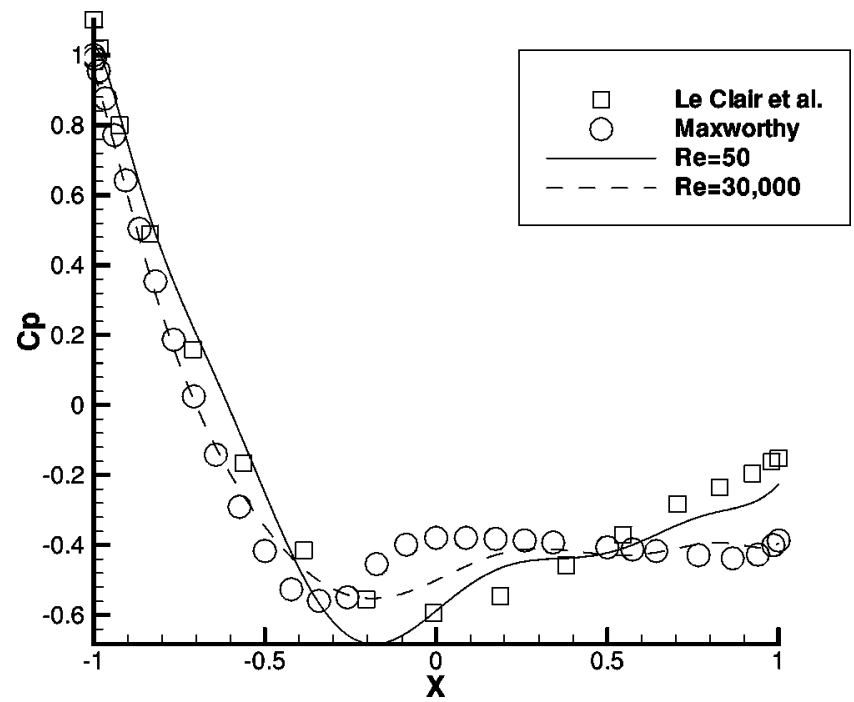

FIG. 3. Comparisons of computational $C_{p}$ results with literature data for the laminar flow cases. The curves are the computational results and the symbols are the literature data.

and 0.125 in volume II in the $x$ direction. In the $y$ - and $z$ directions, the same grid ratio was used as in the laminar cases. After the projection, the number of grid points on the surface of the sphere was increased to 3189 in the flow direction and 96 in the azimuthal direction. In addition to using the same velocity and continuity convergence criteria as in the laminar cases, a convergence residual for the turbulent viscosity was set at $10^{-3}$.

In the following comparisons, for laminar cases a Reynolds number of $3 \times 10^{4}$ was selected since reputable experimental data at this Reynolds number can be found in Maxworthy (1969). A low Reynolds number case of $\mathrm{Re}=50$ was also added to compare with literature data for low Reynolds number cases where vortex shedding does not exist in the wake. For turbulent cases, very high Reynolds number cases measured by Achenbach (1972) have been found at Reynolds numbers of $1.59 \times 10^{5}, 5.7 \times 10^{5}$, and $2.5 \times 10^{6}$.

Figures 3 and 4 show comparisons between the computational results and literature data for $C_{p}$ versus $x$ on the surface of a sphere. Two sets of data have been compared for the laminar cases in Fig. 3: one at $\mathrm{Re}=50$ by Le Clair et al. (1970) and the other at $\operatorname{Re}=3 \times 10^{4}$ by Maxworthy (1969). (It should be noted that the Reynolds number specified here was based on radius, while most of the published literature uses diameter.) Figure 3 shows that the agreement is good, particularly at the upstream half of the sphere before the adverse pressure gradients occur. Three turbulent cases are shown in Fig. 4 for comparison with Achenbach's experimental results. Again, good agreement at the upstream half of the sphere was achieved. For the two highest Reynolds number cases, the trough value and the rebounce of the $C_{p}$ were slightly underpredicted in the simulations. This may be attributed to the turbulence model used in the simulations, or the resolution issues near the solid boundary. However, it was not the intention of this paper to resolve the turbulent simulation issues and, as stated previously, the primary interest was within the laminar flow regime. Nevertheless, the good agreement between calculation and measurement pro-

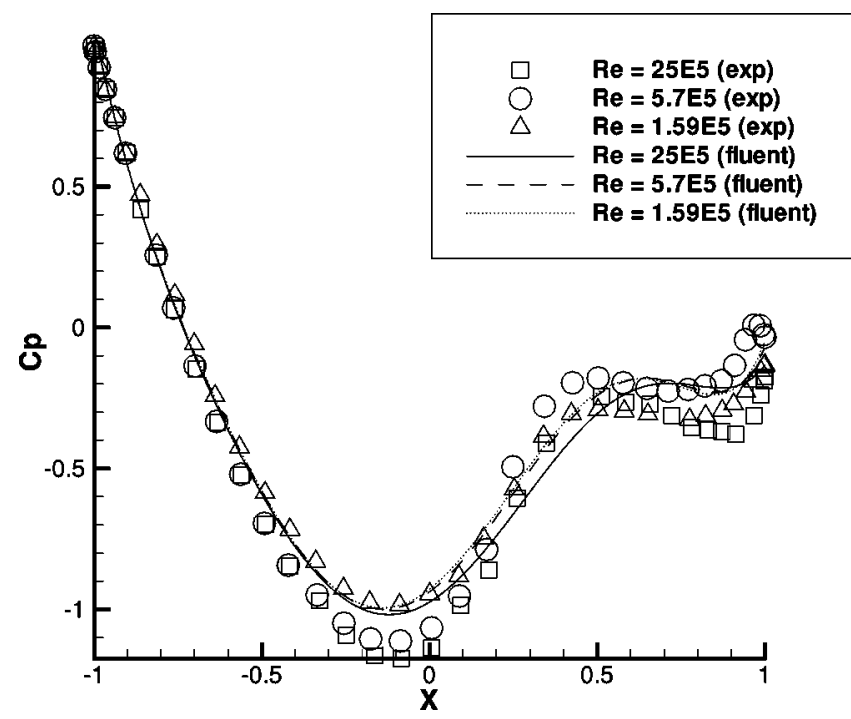

FIG. 4. Comparisons of computational $C_{p}$ results with literature data for the turbulent flow cases. The curves are the computational results and the symbols are the literature data.

vides evidence that this computational tool could be used for further investigation, when experimental data associated with more complicated windscreen materials such as porous foams are difficult to obtain.

Figure 5 shows a plot of the WNR versus Reynolds number. The calculated WNR data results from the computational model are shown along with that from experimental measurements. A correlation relationship was obtained by curve-fitting these data. The restrictions on the behavior at the two extremities were also applied to the correlation; i.e., $\mathrm{WNR}=\infty$ and $6.02 \mathrm{~dB}$ at $\operatorname{Re}=0$ and $\infty$, respectively. The resulting correlation was of the form

$$
\mathrm{WNR}=\alpha \mathrm{Re}^{-\beta}+6.02,
$$

where $\alpha=18.057$ and $\beta=0.171$. This curve shows that when the Reynolds number decreases the wind noise reduction increases, until it reaches an extreme condition where reduc-

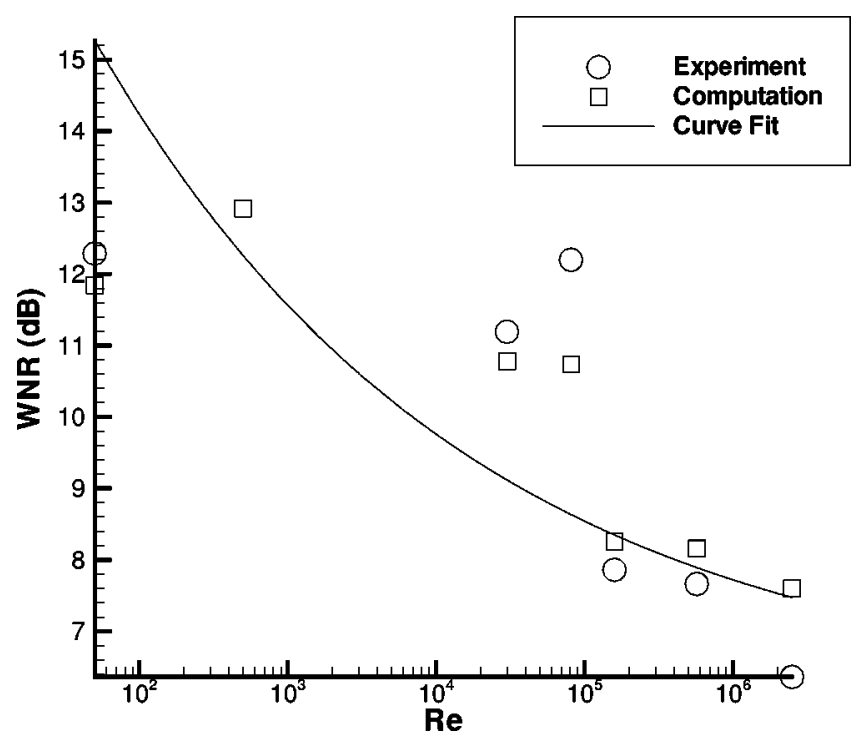

FIG. 5. Correlation between Re and WNR. The curve is from curve fitting of the literature experimental data and our computational data. 
tion is sufficiently large such that the noise becomes zero at the center of the sphere at very low Reynolds number.

It should be noted that the experiments in Morgan (1993) used a Pitot tube embedded in 90-mm and 180-mmdiameter windscreens made of foam material. These windscreens were neither impermeable nor rigid. The corresponding Reynolds numbers for a free-stream velocity of $9.5 \mathrm{~m} / \mathrm{s}$ associated with these measurements were $2.93 \times 10^{4}$ and $5.86 \times 10^{4}$, respectively. The experimental data showed that the $C_{p}$ value at the stagnation point was only 0.8 for each of the two cases. However, the integration of the $C_{p}$ distribution data still gave the WNR values of 16.3 and $14.6 \mathrm{~dB}$, respectively, for the $90-\mathrm{mm}$ and $180-\mathrm{mm}$ windscreens, which were close to the measured reductions. These WNR values are higher than those given from Fig. 5, which are approximately 9.2 and $8.8 \mathrm{~dB}$, respectively, for these two Reynolds numbers. These differences are apparently due to the increased wind noise reduction capability offered by the foam materials used in the experimental tests. If one revisits Fig. 1, which contains the direct measurement data for WNR, the corresponding Reynolds numbers of the two cases in Fig. 1 are approximately $1.5 \times 10^{4}$ and $3.0 \times 10^{4}$ for the $90-\mathrm{mm}$ and 180-mm windscreens, respectively. At these two Reynolds numbers, the WNR from Fig 5 is $9.5 \mathrm{~dB}$ for the $90-\mathrm{mm}$ case and $9.2 \mathrm{~dB}$ for the $180-\mathrm{mm}$ case. Such a small difference cannot be discerned in Fig. 1, where the slight increase of WNR with rising screen number is the dominant trend. For high Reynolds numbers $\left(\operatorname{Re}>10^{4}\right)$, Fig. 5 shows that a Reynolds number increase of ten times causes only less than 1 dB WNR decrease. Therefore, WNR is not very sensitive to changes in Reynolds number at the high Reynolds number range. This correlation is only for low-frequency wind noise reduction, because this paper is only concerned with wind noise below $10 \mathrm{~Hz}$ where it is independent of the frequency (as shown in Fig. 1). Higher-frequency turbulence will require a more detailed study of the unsteady flow. Unsteady flow over a sphere has been investigated in recent literature such as Mei and Adrian (1992), Tomboulides and Orszag (2000), Howe et al. (2001), among others.

\section{CONCLUSIONS}

The conclusions here only apply to the model windscreen in this analysis, which is impermeable and rigid, yet of no impedance to pressure fluctuation transmission. This model has shown promising comparisons with experiments and has the potential of being adopted for more complicated windscreens. For low-frequency turbulence, wind noise reduction is independent of frequency; therefore, steady-state flow analysis can be used to determine wind noise reduction levels. It is shown in this paper that for a spherical windscreen, the value of the noise reduction at the center can be calculated from integration of the surface pressure coefficient on the sphere in a corresponding steady-state flow. By using computational fluid dynamic simulations, and reprocessing experimental measurement data in the literature in a wide range of Reynolds numbers, it has been found that, although it is independent of frequency, wind noise reduction is correlated with the Reynolds number defined by the wind speed and the windscreen radius. A power-fit type correlation shows that the wind noise reduction increases with decrease of the Reynolds number, with the lowest extreme of $6.02 \mathrm{~dB}$ in the inviscid flow case and a value of infinity in the creeping flow case.

\section{ACKNOWLEDGMENTS}

The authors are grateful for fruitful discussions with Dr. R. Raspet and Dr. J. Seiner of National Center for Physical Acoustics (NCPA) at University of Mississippi, and the partial financial support from NCPA. Special thanks go to Dr. Raspet for his comments on an earlier version of the manuscript. The authors would also like to thank the Associate Editor and the reviewers for a number of insightful comments.

Achenbach, E. (1972). "Experiments on the flow past spheres at very high Reynolds numbers," J. Fluid Mech. 54, 565-575.

Currie, I. G. (1993). Fundamental Mechanics of Fluids, 2nd ed. (McGrawHill, New York)

Hosier, R. N., and Donavan, P. R. (1979). "Microphone windscreen performance," National Bureau of Standards Report, NBSIR 79-1599.

Howe, M. S., Lauchle, G. C., and Wang, J. (2001). "Aerodynamic lift and drag fluctuations of a sphere," J. Fluid Mech. 436, 41-57.

Jackson, J. D. (1963). Classical Electrodynamics (Wiley, New York).

Le Clair, B. P., Hamielec, A. E., and Pruppacher, H. R. (1970). "A numerical study of the drag on a sphere at low and intermediate Reynolds numbers," J. Atmos. Sci. 27, 308-315.

Lee, S. (2000). "A numerical study of the unsteady wake behind a sphere in a uniform flow at moderate Reynolds numbers," Comput. Fluids 29, 639667.

Maxworthy, T. (1969). "Experiments on the flow around a sphere at high Reynolds numbers," J. Appl. Mech. E36, 598-607.

Mei, R., and Adrian, R. J. (1992). "Flow past a sphere with an oscillation in the free-stream velocity and unsteady drag at finite Reynolds number," J. Fluid Mech. 237, 323-343.

Morgan, M. S. (1993). "An investigation of the sources and attenuation of wind noise in measurement microphones," Ph.D. dissertation, Department of Physics and Astronomy, University of Mississippi. PARGUM Report 93-01.

Morgan, S., and Raspet, R. (1992). "Investigation of the mechanisms of low-frequency wind noise generation outdoors," J. Acoust. Soc. Am. 92, $1180-1183$.

Patankar, S. V. (1980). Numerical Heat Transfer and Fluid Flow (McGrawHill, New York).

Phelps, W. D. (1938). "Microphone wind screening," in RCA Review 3 (Radio Corporation of America, New York), pp. 203-212.

Spalart, P., and Allmaras, S. (1992). "A one-equation turbulence model for aerodynamic flows," AIAA Paper No. AIAA-92-0439, American Institute of Aeronautics and Astronautics, 1992.

Strasberg, M. (1988). "Dimensional analysis of windscreen noise," J. Acoust. Soc. Am. 83, 544-548.

Tomboulides, A. G., and Orszag, S. A. (2000). "Numerical investigation of transitional and weak turbulent flow past a sphere," J. Fluid Mech. 416, $45-74$. 